Results A total of 384 infiltrations were recorded. The average monthly rate pre-intervention was 26.8 and post-intervention was 20.7 with a p-value of 0.04 (statistically significant). Although a reduction was seen post-intervention, there was a rebound in infiltrations starting Nov 2010 to Feb 2011, which was recognized at the time, attributed to a high turnover of nurses and higher rates of admissions. We repeated a series of in-services with a subsequent reduction in infiltration rate.

Conclusions The overall pattern shows a statistically significant reduction in infiltration rate after the intervention. However, continuous surveillance and reinforcement of interventions is mandatory to sustain improved rates.

\section{USE OF THE MODIFIED BRIGHTON PEDIATRIC EARLY WARNING SCORE (PEWS) IN A NORWEGIAN DEPARTMENT FOR CHILDREN AND ADOLESCENTS}

doi:10.1136/archdischild-2012-302724.1565

EH Eggen, A Solevåg, J Schrøder, B Nakstad. Dept. of Pediatrics, Akershus University Hospital, Lørenskog, Norway

Background and Aims Structured observations and examination are crucial. However, paediatric early warning scoring systems is a relatively new concept.

We aimed to investigate the feasibility of a modified version of the Brighton paediatric early warning score (PEWS) in our department.

Methods The PEWS consists of respiratory, circulatory and behavioural parameters with $0-3$ points assigned for each category. Persisting postoperative vomiting and continuous inhalation medications give 2 extra points each. Hence, a score of 0 to13 can be assigned, score 0 being most favorable.

All acutely referred children in April/May 2011 were scored.

Patients were retrospectively categorized into diagnose groups. We included patients with score $0(n=89)$ and those with scores $\geq 4(n=49)$. Results The 0 group:

Sixty percent of patients considered to be well enough to be sent home without admittance to the ward had a PEWS of 0 . Only $10 \%$ of patients that were admitted had a PEWS of 0 . Allergic reactions (excl. anaphylaxis), arthritis, vasculitis, abnormal head circumference, psychosomatic disorders, constipation and upper respiratory tract infection typically gave low PEWS.

The $\geq 4$ group:

Diagnoses like asthma, bronchiolitis and other lower respiratory tract infections gave almost invariably high PEWS. In addition, cardiological conditions gave PEWS $\geq 4$.

PEWS scoring varied with age as 18/35 (60\%) of $0-2$ year olds scored $\geq 4,11 / 20(55 \%)$ at 2 years and only $17 / 83(20 \%)$ of patients $>3$ years scored $\geq 4$.

Conclusions The modified PEWS may be a useful method to detect high-risk patients in our department.

\section{PARENTS/CAREGIVERS' KNOWLEDGE TOWARDS MEDICINES ADMINISTRATION IN PAEDIATRICS}

doi:10.1136/archdischild-2012-302724.1566

C Teixeira de Barros, TM Salgado. iMed.UL - Research Institute for Medicines and Pharmaceutical Sciences, Faculty of Pharmacy, University of Lisbon, Lisbon, Portugal

Background and Aims The increasing complexity in paediatric patient care emphasizes patient safety as a topic of high priority. Parents/caregivers' lack of knowledge on how to administer extemporaneous formulations in paediatrics can be a potential source of medications errors.

Methods A 2-month cross sectional study was conducted with a convenience sample of paediatric outpatients' parents/caregivers from four hospitals in Lisbon. A questionnaire was developed to assess knowledge on how to administer the medicine (liquid or powder), how to measure the dose to be administered, administration schedule, storage conditions, validity period of the extemporaneous formulation, and adequate behaviour in case of missing a dose or vomiting immediately after taking the medicine. A univariate analysis was performed using SPSS v.19.

Results Eighty-four individuals participated in the study. The mean (SD) age was 34 (18.6) years, 26.0\% were non-Caucasian, $75.3 \%$ were married, $46.8 \%$ had an average of nine years of education and $50.6 \%$ were professionally active.

The mean level of knowledge as assessed by the questionnaire was $53.7 \%$. The lowest levels of knowledge were found for adequate behaviour in case of missing a dose or vomiting after taking the medicine, for which only $10.7 \%$ and $20.2 \%$ parents/caregivers, respectively, gave the correct answer. Non-Caucasian parents/caregivers and lower education level were significantly associated with a deficit of knowledge $(p<0.05)$.

Conclusions Low levels of knowledge were found among parents/ caregivers of paediatric patients. Strategies to enhance knowledge should be developed to improve patient safety.

\section{A REVIEW OF PAEDIATRIC HANDOVERS - ARE THEY SAFE AND EFFECTIVE?}

doi:10.1136/archdischild-2012-302724.1567

IP Nath, 'P Desai, ${ }^{2}$ W Kelsall. 'Neonatal Unit, Ipswich Hospital NHS Trust, Ipswich; ${ }^{2}$ Neonatal Unit, Cambridge University Hospitals NHS Foundation Trust, Cambridge, UK

Background With changing work patterns effective handovers are essential for patient safety and continuity of care. Handovers also provide educational opportunities, to initiate or complete work place based assessments (WPBA), improve communication, management skills to prioritise work.

Methods A 10 point online questionnaire was sent by email to all the trainees and tutors from June - August 2010.

Results Responses were received from 17 trusts (17/18 hospitals, 94\%) and from 56 trainees (18 ST1-3 and 38 ST4-8). Feedback was received on a total of 73 handovers: $36 \%$ covered both general paediatrics and neonates; $27 \%$ general paediatrics only and $23 \%$ neonatal intensive care. $78 \%$ of respondents were involved in two or more handovers during the working day. $69 \%$ of the handovers were lead by consultants or registrars. All the handovers had registrars present, $93 \%$ had junior trainees, $82 \%$ consultants and $34 \%$ had members from nursing team. $69 \%$ of the handovers were conducted with the aid of printed sheets. Only $51 \%$ of the handovers started on time, $19 \%$ were free from distractions by allied professionals and just $4 \%$ were 'bleep' free. $64 \%$ had some educational activity within the time allocated. WPBAs were initiated or completed in only $3 \%$ of handovers.

Conclusions Handover practice varies between hospitals. Most are well organised but many do not start on time and are not free from interruptions. Formal teaching was not a regular feature of handovers and WPBA were rarely initiated or completed. The role of the handover in training could be developed further.

\section{BURTON NEONATAL TRACK AND TRIGGER OBSERVATION CHART}

doi:10.1136/archdischild-2012-302724.1568

${ }^{1}$ M Ahmed, ${ }^{2}$ Phillips, ${ }^{1}$ A Manzoor. 'Paediatrics; ${ }^{2}$ Midwifery Sister, Burton Hospitals NHS Foundation Trust, Burton Upon Trent, UK

Background The use of early warning system scores and track \& trigger charts is widespread in adult and paediatric hospitalised patients. Its use in neonatal group is not well recognized. Lack of well established normal ranges for biophysical variables in preterm/ term neonates illustrate difficulties in establishing a scoring system 